\title{
Treatment of Secretory Otitis Media by Wind Based on the Relationship Between Lung and Ear
}

\author{
Manli Wang ${ }^{1, *}$, Xiong Zhang ${ }^{2}$ \\ ${ }^{1}$ Shaanxi University of Traditional Chinese Medicine, Xianyang 712046, Shaanxi, China. \\ ${ }^{2}$ Affiliated Hospital of Shaanxi University of Traditional Chinese Medicine, Xianyang 712046, Shaanxi, China.
}

\begin{abstract}
How to cite this paper: Manli Wang, Xiong Zhang. (2021) Treatment of Secretory Otitis Media by Wind Based on the Relationship Between Lung and Ear. International Journal of Clinical and Experimental Medicine Research, 5(1), 15-18.

DOI: 10.26855/ijcemr.2021.01.004
\end{abstract}

Received: December 6, 2020

Accepted: December 30, 2020

Published: January 8, 2021

*Corresponding author: Manli Wang, Shaanxi University of Traditional Chinese Medicine, Xianyang 712046, Shaanxi, China.

\begin{abstract}
Secretory otitis media is a non-suppurative inflammatory disease of middle ear characterized by conductive deafness and tympanic effusion. The etiology and pathogenesis of secretory otitis media are still not very clear, among which the theory of negative pressure of middle ear and the theory of infection are recognized by most scholars. According to the theory of negative pressure of middle ear, secretory otitis media is caused by poor function of eustachian tube, poor gas exchange of middle ear mucosa or bad vaporization of mastoid process, and dysfunction of cleaning function of middle ear, which leads to negative pressure of middle ear, then mucosal edema and exudation, which leads to accumulation of middle ear fluid and formation of secretory of middle ear inflammation. Otitis media will be affected by the progress of secretory otitis media is serious. If the treatment is not timely, it can develop into adhesive otitis media. At present, the treatment of the disease is mostly aimed at antibacterial and liquid excretion. The drug treatment has surface active substances, corticosteroids, antibiotics, antihistamines and so on, but its curative effect is not accurate, easy to repeat, and has different degrees of adverse reactions. Therefore, the treatment of secretory otitis media from the perspective of traditional Chinese medicine has been paid more and more attention. Under the guidance of the theoretical system of "holistic concept" and "dialectical treatment" in TCM clinic, it is helpful to further apply it in clinic and get better therapeutic effect.
\end{abstract}

\section{Keywords}

Secretory otitis media, lung and ear, wind medicine

\section{Understanding of secretory otitis media in TCM}

Secretory otitis media belongs to the category of "ear distention and ear closure" in traditional Chinese medicine. Ear distention is the beginning of the disease. It is mainly caused by the feeling of stuffy distension in the ear, or both pain, which is caused by wind evil, so it is also called "wind deafness". The ear is closed for a long time, the ear is blocked, the clear orifices are blocked, the hearing is obviously decreased, most of them are repeated attacks of ear distension, the evil poison is retained, and the delay is long, so there is also the name of "Qi closed deafness", which is called. The Sui Dynasty's Nao Yuan Fang's “On the Pathology of All Diseases” records: "Wind into the ear pulse, so that the Qi ruffian, so the wind deaf”. Ear distension is due to exogenous wind evil, lung loss and decline, along the meridian, evil closed ear orifices; or wind evil attack, spread in Shaoyang, along the meridian against the ear, closed obstruction clear orifices. Ear closure due to ear distention or repeated attacks caused by evil 
poison retention, phlegm turbid stasis blocking ear orifices, because of the obstruction of the eustachian tube phlegm dampness stasis accumulated in the middle ear cavity, there is tympanic effusion sign. Therefore, Chinese medicine believes that secretory otitis media is mainly wind evil invading the lung, affecting the function of lung propaganda and lowering, leading to water and dampness stagnation, so using sparse wind to promote lung as the basic treatment principle, and to make lung qi clear [2].

\section{Theory of Treating Ear Disease from Lung}

2.1 In the meridian collateral relationship: the hand Taiyin lung meridian collaterals in the ear. Pinch nose drum qi, Qi runs through the ear, indicating that lung qi and ear. Lingshu xie Qi zang-fu organs disease shapes points “Twelve meridians, 365 collaterals, its blood gas on the face of the empty orifices... Don't be angry and listen”. On Su Wen Miao Ci contains "evil influence in the hands and feet less Yin, Tai Yin, foot Yangming collaterals... These five collaterals are in the ear, and the left frontal angle of the collaterals is also", indicating that the collaterals of the lung enter directly into the ear, and the lungs and ears are related through the collaterals. Warm Jingwe points out that "lung knot point in the ear, known as caged scallions, the main listening". Professor Gan Zuwang, a famous doctor, believes that "listening" refers to the auditory function of the ear. And among them, "LongCong" is the meridian qi of lung meridian in ear [3].

2.2 In the physiological relationship: lung qi, lung qi through the ear, and lung and kidney gold and water, so lung and ear function is closely related. In Zhengzi Huibu records: "The kidney opens the orifices to the ear, the ear can listen to the sound, the lung also. Because of the main lung Qi, a body of Qi in the ear is also". Li Dongyuan put forward in the Theory of Spleen and Spleen and Stomach On the Interaction of Qi in the Five Viscera". It is the body of the kidney and the use of the lung that the ear is connected with the weather and the orifices of the kidney. Besides the main lung, the main propaganda speed drops, flies up to fill, the upper coke development, if the fog dew, the cloth Qi and blood body fluid, in order to nourish the empty orifices, the normal physiology of the ear depends on this.

2.3 The pathological relationship: (1) Lung qi is impassable, cannot penetrate the ear. Zaijin You in Medical Books records: "Deafness to treat the lung, is the lung meridian wind heat, phlegm and saliva stagnation syndrome, lung collaterals will be in the ear, its qi impassable, so deafness, so it is appropriate to treat its lungs, so that Qi operation is deafness cured". It is common in clinic of wind evil invades lung, lung qi cannot be purged and cause ear distention pain, ear blockage, tinnitus and deafness and other diseases [3]. (2) Lack of lung qi cannot penetrate the ear. SuWen On the time og gas accumulation records: "Lung disease... Deficiency is less qi cannot be reported, deafness and dry pharynx". Standards of Diagnosis and Treatment points "deficiency of Qi energy will be hyperactivity of cold. Ear of Zong Qi, the lack of Lung Qi will cause desfness”. That is, deficiency of lung qi, lung a hundred veins, a hundred veins deficiency, will lead to a deficiency of the ear vein, vulnerable to external evil invasion, and prone to ear stasis, so that auditory conduction is blocked, so deafness.

\section{The role of wind evil in the pathogenesis of secretory otitis media}

Plain Questions the theory of the wind says that "wind being primary pathogen". The wind evil for the disease at all seasons, and wind evil with cold evil often will co-occur. In addition, the cold evil causes contraction. If the evil of cold and cold attacks, lung disease through, can make the ear inside the gas PI Plug cannot vent, so there is a feeling of ear blocage and hearing loss. If the ear obstruction, long illness into the meridians, poison retention for a long time, Qi snd blood stasis ear obstuction. Then the eardrum loses normal luster, thickens, or ensnared adhesion, or gray calcified spots, hearing gradually decreases. Therefore, the wind attack, plug the ear orifice is the basic pathogeneis of ear distension and closure. The SunWen Tai Yin-yang theory: "Injury in the wind, the upper part of the body being first attacked”. The characteristic of wind evil is blown up scattered. It has the characteristics of rising, upward and outward. Diseases caused by wind evil vulnerable upper part, the lungs are the canopy of the viscera, the lungs are the highest in the viscera. Wind evil hurts people. The lungs besr the brunt. "The lung gas penetrates the ear. If the lung is harmonious, the ear can be unblocked”. Only after the lung Qi is harmony, obsessed with the ear through. If the wind evil attacks the lung, the lung loses its prostration. Then appears ear distending pain, ear block and stuffy feeling, tinnitus and deafness, etc. Wind evil is Yang evil. The skin of the Yang part of the human body is prone to injury. The mucous membrane and cila on the inner surface of the middle ear belong to the fur. It is easy for wind evil to enter from skin and mucous membrane, and the wind attack appearance. Wei Qi does not declare, affect lung main Qi function. And then lead to auricular-orifice. Blockage occues with distension, sense of fullness and occlusion. The announcement and purge of lung qi are abnormal, and the function of regulating channel is abnormal, which leads to hydro-fluid stop of auricular-orifice. 


\section{Shufeng Xuanfei-The use of wind medicine}

Mingjie Wang [4] believes that wind medicine is the most commonly used and effective type of Xuanfu drugs. The concept of wind medicine is first found in Li Dongyuan's Theory of spleen and stomach, but it is not clearly defined. Xu Dachun put forwards: "Any medicine of light quality and Qi Sheng, all belong to wind medicine”. Later, doctors extended the concept of wind medicine, and believed that the drugs with the functions of removing wind and removing dampness, dispelling wind and dredging collaterals all belong to the category of wind medicine [5]. The flavor of wind and medicine is light and thin, the medicine is rising and floating, the rise is clear and light, it has the characteristics of rising hair, adjusting to reach, comfortable and so on. Therefore, "Shufeng Xuanfei" is the basic treatment of secretory otitis media, the use of wind medicine throughout the treatment, in order to achieve the effect of Tongli ear orifices, the treatment should be based on clinical syndrome differentiation, supplemented by solid surface, dehumidification, blood stasis, can achieve good results: (1) Shufeng GuBiao: Plain body lung qi deficiency, Wei table is not solid, loose, wind-cold evil often take advantage of the deficiency, lung meridian disease, ear through Qi ruffian plug does not declare. Therefore, it can be seen that the sense of blockage in the ear, hearing loss, a finger pressing the ear door, can help dredge the meridian qi, reduce the symptoms of ear discomfort. Lack of lung qi camp guard discord, often accompanied by nasal congestion, sweating, bad wind, afraid of cold. Treatment with wind-sparing with warm lung solid table, it often on the basis of wind medicine to choose Astragalus, Fangfeng, Atractylodes macrocephala and so on. (2) QuFeiChuShi: The most important physiological function of the lung is to promote and purge. The lung is the source of water. Its function is to nourish the skin and viscera, and to keep the balance of water and fluid metabolism in order to maintain the balance of water and fluid metabolism. Its function is to nourish the skin and viscera, and to keep the balance of water and fluid metabolism in order to maintain the balance of water and fluid metabolism. If the water channel is dereliction of duty, causing the water and liquid to stop gathering the ear orifices, it will be seen that the ear is more stuffy. The tympanic membrane was found to be reddish, invaginated or with liquid plane. The lung and spleen and stomach cooperate closely with each other in transporting fine and dispersing water. Therefore, it can be accompanied by loose stools and other spleen deficiency, fat tongue with teeth marks, white fur greasy, weak pulse. The Huangdi Neijing records: "Central wet, wet raw soil... Wind wins dampness", wind medicine through its dispersing nature, can speed up the dissipation of dampness evil, disease recovery. We often choose Fangfeng, Atractylodes, Notopterygium on the basis of wind medicine. (3) QuFengHuaShi: Evil attacks on ear orifices can easily lead to choroid block, Qi movement and movement is not smooth. "Qiis the leader of blood, Qi runs smoothly and blood runs through". Qi and blood flow is restricted to form blood stasis, can be seen in the ear stuffy clogging feeling obvious, not cured for a long time, even such as material barrier, hearing loss gradually. It has the function of regulating the circulation of blood vessels, activating blood circulation and strengthening the function of removing blood stasis. At the same time, compatible with the use of drugs to promote blood stasis, dispelling the invading wind-evil to get rid of the effect of going out, often on the basis of wind-drug plus salvia miltiorrhiza, Chuanxiong, Angelica sinensis and so on [6].

\section{Example}

Yang Mou, 54 years old, first visit on April 5, 2020. Main complaint: left ear distention and blockage feeling 2 months. The patient complained that before 2 months ago because of the cold after the first out of the left ear distention stuffy feeling, hearing loss, on their own in the local clinic to see a doctor, given oral medicine "Gankang, cefixime capsule", after taking the symptoms did not alleviate. He went to the people's Hospital of XX county to see a doctor, to do an ear endoscopy and acoustic impedance examination. Treatment: Antibiotics, hormones, mucus-promoting agents and other drugs were not effective, and then the feeling of left ear distention and blockage gradually aggravated, so we went to our outpatient clinic, hoping to use traditional Chinese medicine treatment. Present symptoms: Left ear distention and blockage, hearing loss, nasal congestion and runny nose, slightly poor stomach, sometimes stomach distension, stool is not shaped, facial color is not Hua, urine is not yellow, night rest is poor, tongue is light, fur is thin white, edge has tooth print, pulse is weak. Special examination showed that the left ear tympanic membrane was orange, invaginated, nasal endoscopy: bilateral inferior turbinate slightly hypertrophy, no abnormal secretion in the middle nasal canal, nasal septum deviation to the right, smooth nasopharyngeal mucosa, bilateral pharyngeal recess symmetry. Supplementary examination: (1) Pure tone electrical audiometry: left auditory threshold :125 Hz, 250Hz, 500Hz, $1000 \mathrm{~Hz}, 2000 \mathrm{~Hz}, 4000 \mathrm{~Hz} 65 \mathrm{~dB}, 60 \mathrm{~dB}, 55 \mathrm{~dB}, 60 \mathrm{~dB}, 55 \mathrm{~dB}, 65 \mathrm{~dB}$., respectively Bone conduction is generally normal, right ear hearing is normal; (2) Acoustic impedance diagram: the left ear is C and the right ear is AS. Western medicine diagnosis: chronic secretory otitis media. Diagnosis of TCM: Ear closure. Patients due to exogenous wind evil, coupled with work reasons, improper diet, often hunger or full- 
ness, resulting in the abnormal function of the upper abodomen spleen and stomach. On account of Spleen weakness. The function of transporting water liquid is abnormal, resulting in Qing-Yang not rising, Zhuo-Yin not falling, water dampness blocking ear orifices and ear closing disease. The patients' ear distention and tightness were more obvious, and appetite reduction or bad appetite, sometimes the stomach bilges, shapeles stool, lusterless complexion, pale tongue with white and tooth prints, weak pulse. All of these are symptoms of water retention due to hypofunction of the spleen. Therefore, the treatment of dispelling wind and dehumidification, invigorating spleen and opening orifices as the method, the prescription of LingGuiZhuGan flavor treatment. Poria cocos 30g, Astragalus 15g, Magnolia 12g, Atractylodes macrocephala 12g, Atractylodes macrocephala 12g, Fangfeng 10g, Guizhi 10g, igusticum chuanxiong 10g, Acorus calamus 9g, Glycyrrhiza uralensis 6g. A total of 7 doses, 1 dose, morning and evening, water decoction. At the same time, the middle ear negative pressure therapy instrument treatment, once a day. When the second diagnosis, the patient consciously left ear distention and blockage relief, hearing slightly improved, electro-earoscopy examination: left ear tympanic membrane pale red, slightly trapped, continue to follow the treatment ahead, after 2 weeks of treatment, the patient consciously left ear distention and stuffy plug feeling basically disappeared, hearing basically returned to normal. Electrocardiogram examination: left ear tympanic membrane is complete, no hyperemia, recheck electric audiometry and acoustic impedance examination, electric audiometry examination: left ear air conduction threshold :125 Hz, $250 \mathrm{~Hz}, 500 \mathrm{~Hz}, 1000 \mathrm{~Hz}, 2000 \mathrm{~Hz}, 4000 \mathrm{~Hz}$ respectively $30 \mathrm{~dB}, 35 \mathrm{~dB}, 30 \mathrm{~dB}, 35 \mathrm{~dB}, 25 \mathrm{~dB} 255 \mathrm{~dB}$, bone conduction is normal. Acoustic impedance examination: the binaural tympanic charts are all A type. No recurrence was found in follow-up for 2 months. Reuse Poria cocos to strengthen spleen and dampness to stop diarrhea. Astragalus tonifying lung and spleen, promoting water discharge pus. Magnolia and Angelica dahurica are scattered wind to drain pus, Xanthium to clear the lungs, cold wind, Atractylodes macrocephala invigorate spleen and invigorate qi, dry dampness and water, radix sileris Dispelling wind and dampness and relieving itching, Guizhi warming Yang to help transport water, Chuanxiong Xin San Wen Tong, Acorus calamus seeps through the orifices, making the eustachian tube unobstructed. The above-mentioned Chinese medicine combined to achieve dispelling wind and dehumidification, invigorating spleen and orifices.

In a word, based on the relationship between lung and ear, the treatment of secretory otitis media from wind theory, taking "sparse wind and promoting lung" as the basic treatment principle, according to clinical syndrome differentiation and application of wind medicine, the treatment of secretory otitis media with sparse wind, dispelling wind and dehumidification, dispelling wind and blood stasis is effective, which can give full play to the advantages of traditional Chinese medicine in the treatment of secretory otitis media, and is worth popularizing.

\section{References}

[1] Chen, L. (2001). The etiology and pathogenesis of secretory otitis media. Division of Otolaryngology, Foreign Medicine, 2001, 25(3): 135.

[2] Dong, H., Honghua Lu, Ming Hu, Guangai Hu. (2003). "Discussion on the Basic Theory of Western Medicine on the Treatment of Oculi (ear) Disease from the Lung” [J]. Chinese Journal of Otorhinolaryngology, Integrated Chinese and Western Medicine, 2003, 11(4): 157.

[3] Yifei Chen. (2006). A Brief Discussion on "Deafness and the Treatment of Lungs” [J]. Gansu Traditional Chinese Medicine, 2006, 19(11): 16-17.

[4] Mingjie Wang, Shufen Huang, Zaiqiong Luo, et al. (2011). New knowledge of wind and medicine [J]. Journal of Luzhou Medical College, 2011, 34(5): 570-572.

[5] Weiying Zhang, Junfu Hu, Jian Zhong. (2019). Application of wind medicine in chronic glomerulonephritis [J]. World’s Latest Medical Information Digest, 2019, 19(46): 82-83.

[6] Yue Li, Xu Wang. (2019). On the Treatment of Allergic Rhinitis Based on the Theory of Nasal Xuanfu Guangxi Traditional Chinese Medicine, 2019, 10, 42(5): 52. 